\title{
Scattering Length Scaling Laws for Ultracold Three-Body Collisions
}

\author{
J. P. D'Incao and B. D. Esry \\ Department of Physics, Kansas State University, Manhattan, Kansas 66506
}

\begin{abstract}
We present a simple picture that provides the energy and scattering length dependence for all inelastic three-body collision rates in the ultracold regime for three-body systems with short range two-body interactions. In particular, we present the scaling laws for vibrational relaxation, threebody recombination, and collision-induced dissociation for systems that support $s$-wave two-body collisions. These systems include three identical bosons (BBB), two identical bosons (BBB'), and two identical fermions $\left(\mathrm{FFF}^{\prime}\right)$. Our approach reproduces all previous results, predicts several others, and gives the general form of the scaling laws in all cases.
\end{abstract}

PACS numbers: $34.10 .+\mathrm{x}, 32.80 . \mathrm{Cy}, 05.30 . \mathrm{Jp}$

The use of external magnetic fields to control the atomic interactions in trapped ultracold quantum gases has proven to be an extraordinary tool to explore different quantum regimes. At low temperatures, only the two-body $s$-wave scattering length $a$ is needed to characterize the atomic interactions and can assume practically any value from $-\infty$ to $+\infty$ by tuning a magnetic field near a diatomic Feshbach resonance. This tunability has been used, for instance, to convert fermionic atoms into weakly bound bosonic molecules which, in some cases, were remarkably long lived [1]. In the quantum degenerate regime, this system provides a unique opportunity to explore the crossover between a Bose-Einstein condensate (BEC) of molecules and the BEC of atomic Cooper pairs, the BEC-BCS (Bardeen-Cooper-Schrieffer) transition.

Since the molecular lifetimes are influenced by threebody processes, these experiments underscore the importance of knowing the dependence of ultracold threebody collision rates on $a$. In particular, vibrational relaxation, $\mathrm{X}+(\mathrm{XX})^{*} \rightarrow \mathrm{X}+\mathrm{XX}$, releases enough kinetic energy to free the collision products from typical traps, leading to molecular loss. Collision induced dissociation, $\mathrm{X}+\mathrm{XX} \rightarrow \mathrm{X}+\mathrm{X}+\mathrm{X}$, can also contribute to molecular loss for large $a$. The other possible three-body process, three-body recombination $\mathrm{X}+\mathrm{X}+\mathrm{X} \rightarrow \mathrm{X}+\mathrm{XX}$, contributes to atomic losses. In an experiment, however, the dominant process is determined in part by the threshold and scattering length scaling laws.

Threshold laws, which give the energy dependence for small collision energies, dictate the partial wave that dominates for each process. The threshold law for elastic two-body collisions, for instance, gives an $s$-wave cross section that is constant at threshold while the $l$-th partial wave is suppressed by a factor of $E^{2 l}$. Combined with permutation symmetry requirements, the threshold law thus leads to the familiar conclusion that the collision cross section for two indistinguishable fermions vanishes at low energies. Three-body threshold laws similarly depend on the number and kind of identical particles for each partial wave $J^{\pi}$, where $J$ is the total orbital angular momentum and $\pi$ is the parity [2].

While general results exist for three-body threshold laws, no similarly general scattering length scaling laws have yet been obtained. Specific cases are known: the recombination rate for $\mathrm{B}+\mathrm{B}+\mathrm{B}$ collisions, where $\mathrm{B}$ is a bosonic atom, scales roughly as $a^{4}$ for $0^{+}$and all $a$ [3, 4], and as $a^{8}$ for $2^{+}$and $a>0$ [ 5 . It has also been shown for $a>0$ that the relaxation rate for $\mathrm{B}+\mathrm{BB}$ collisions is linear in $a$ 6], but is $a^{-3.33}$ for $\mathrm{F}+\mathrm{FF}^{\prime}$ collisions 7], where $\mathrm{F}$ and $\mathrm{F}^{\prime}$ are distinguishable fermionic atoms and $a$ is the $\mathrm{F}+\mathrm{F}^{\prime} s$-wave scattering length. This scaling law is qualitatively different from the other known scaling laws and begs the question: how do the three-body processes for other systems scale with $a$ ?

In this Letter we present a simple physical picture within which both the energy and scattering length dependence of all ultracold three-body collision rates (for short-range two-body interactions) can be derived and understood. A simple picture emerges because all threebody systems can be represented by one of four prototype systems (two each for $a>0$ and $a<0$ ). In all cases, though, the rate limiting step is tunneling through a potential barrier in the initial channel. The barrier is determined here from effective three-body potentials obtained from the adiabatic hyperspherical representation [8]. A simple WKB approximation to the tunneling probability is then sufficient to give both the energy and scattering length dependence. Besides their practical use, the scaling laws make explicit the pervasive influence of Efimov physics [9] on ultracold three-body collisions. We restrict our discussion to systems with equal masses and to symmetries that support $s$-wave two-body collisions, representing most cases of experimental interest.

In the adiabatic hyperspherical representation, the three-body effective potentials and couplings are determined from the adiabatic equation [8],

$$
H_{\mathrm{ad}}(R, \Omega) \Phi_{\nu}(R ; \Omega)=U_{\nu}(R) \Phi_{\nu}(R ; \Omega),
$$

where $\Omega$ denotes all hyperangles and $R$ is the hyperradius that, roughly speaking, gives the overall size of the system. The adiabatic Hamiltonian $H_{\text {ad }}$ includes the hyperangular kinetic energy as well as all interactions. By expanding the total wave function on the adiabatic basis 
$\Phi_{\nu}$, the Schrödinger equation is reduced to (atomic units will be used unless otherwise noted):

$$
\left[-\frac{1}{2 \mu} \frac{d^{2}}{d R^{2}}+W_{\nu}\right] F_{\nu}+\sum_{\nu^{\prime} \neq \nu} V_{\nu \nu^{\prime}} F_{\nu^{\prime}}=E F_{\nu} .
$$

In this expression, $\mu$ is the three-body reduced mass, $E$ is the total energy, $F_{\nu}$ is the hyperradial wave function, $V_{\nu \nu^{\prime}}$ is the nonadiabatic coupling responsible for inelastic transitions, and $W_{\nu}$ is the effective potential.

For short range interactions, the asymptotic behavior of $W_{\nu}$ can be derived analytically [10]. In this limit, the molecular channels, which represent atom-molecule scattering, and the three-body continuum channels, which represent collisions of three free particles, are given by

$$
W_{\nu}=E_{v l^{\prime}}+\frac{l(l+1)}{2 \mu R^{2}} \text { and } W_{\nu}=\frac{\lambda(\lambda+4)+15 / 4}{2 \mu R^{2}},
$$

respectively. The molecular bound state energy $E_{v l^{\prime}}$ is labeled by the ro-vibrational quantum numbers $v$ and $l^{\prime}$; $l$ is the relative angular momentum between the molecule and the atom; and $\lambda$ is a positive integer that labels the eigenstates of the hyperangular kinetic energy.

When the $s$-wave scattering length $|a|$ is large, however, an intriguing phenomenon known as the Efimov effect occurs [9], modifying the behavior of $W_{\nu}$. These modifications must be considered in order to properly predict the dependence of the three-body rates on $a$ 3, 4, 5, 6]. In the limit $|a| \gg r_{0}$, where $r_{0}$ is the characteristic size of the two-body potential, Eq. (3) applies only for $R \gg|a|$. For $r_{0} \ll R \ll|a|$, the potentials are proportional to $R^{-2}$, but can now be attractive as well as repulsive. Strictly speaking, the term "Efimov effect" applies only to the emergence of an infinity of three-body bound states for $|a| \rightarrow \infty$. We will instead use the term "Efimov physics" to indicate the qualitative change in behavior exhibited by any system whenever at least two of the three possible $s$-wave scattering lengths is large.

Based on the modifications due to Efimov physics, we can classify all three-body systems into one of two categories: those with an attractive dipole potential for $r_{0} \ll R \ll|a|$ and those without. For equal mass systems, only $0^{+}$bosonic systems fall into the first category. In these systems, the attractive potential appears in the highest vibrationally excited $s$-wave (or weakly bound) molecular channel $\left(l^{\prime}=0\right)$ for $a>0$, and in the lowest continuum channel for $a<0$. The effective potentials for all higher channels are repulsive, but the coefficients differ from those shown in Eq. (3) due to Efimov physics. These effective potentials are conveniently parametrized by,

$$
W_{\nu}(R)=\frac{-s_{0}^{2}-\frac{1}{4}}{2 \mu R^{2}} \text { and } W_{\nu}(R)=\frac{s_{\nu}^{2}-\frac{1}{4}}{2 \mu R^{2}}
$$

The constants $s_{0}$ and $s_{\nu}$ depend on the number of resonant pairs as well as the number of identical particles.

\begin{tabular}{c|lcc|cc}
\hline \hline & $J^{\pi}$ & $l_{\min }$ & $\lambda_{\min }$ & $s_{0}\left(p_{0}\right)$ & $s_{\nu}\left(p_{\nu}\right)$ \\
\hline $\mathrm{BBB}$ & $0^{+}$ & 0 & 0 & 1.0062378 & 4.4652946 \\
& $1^{-}$ & 1 & 3 & 2.8637994 & 6.4622044 \\
& $2^{+}$ & 2 & 2 & 2.8233419 & 5.5082494 \\
\hline $\mathrm{BBB}^{\prime}$ & $0^{+}$ & 0 & 0 & 0.4136973 & 3.4509891 \\
& $1^{-}$ & 1 & 1 & 2.2787413 & 3.6413035 \\
\hline $\mathrm{FFF}^{\prime}$ & $0^{+}$ & 0 & 2 & 2.1662220 & 5.1273521 \\
& $1^{-}$ & 1 & 1 & 1.7727243 & 4.3582493 \\
& $2^{+}$ & 2 & 2 & 3.1049769 & 4.7954054 \\
\hline \hline
\end{tabular}

TABLE I: Coefficients of the potentials in Eqs. (3)- (5). Except for $0^{+}$bosons, the constants correspond to $p_{0}$ and $p_{\nu}$.

For all other cases, including $J>0$, the effective potentials are repulsive in the weakly bound and continuum channels, for $a>0$ and $a<0$. Like the $0^{+}$bosonic case, these potentials are parametrized by constants $p_{0}$ and $p_{\nu}$ :

$$
W_{\nu}(R)=\frac{p_{0}^{2}-\frac{1}{4}}{2 \mu R^{2}} \text { and } W_{\nu}(R)=\frac{p_{\nu}^{2}-\frac{1}{4}}{2 \mu R^{2}} .
$$

In all cases, deeply bound molecular channels are essentially independent of $a$.

The constants $s_{0}, s_{\nu}, p_{0}$, and $p_{\nu}$ can be obtained analytically [9], and numerical values are shown in Table $\square$ for the two partial waves that dominate relaxation and recombination near threshold. Table $\square$ also shows the minimum $l$ and $\lambda$ [see Eq. (31)] allowed by permutation symmetry that, in turn, determine the dominant partial wave for the three-body rates near threshold [2].

The relevant effective potentials and couplings are sketched in Figs. 1(a) and प(b) for $0^{+}$bosonic systems and in Figs. 1(c) and 1(d) for all other cases. For $r_{0}<R<|a|$, the potential curves are given by Eqs. (4) and (5); for $R>|a|$, the curves are those for finite $|a|$ in Eq. (3). The lowest continuum channel, labeled " $\alpha$ ", is the initial channel for recombination. Channel " $\beta$ " is the weakly bound molecular channel and is the initial channel for relaxation. Channel " $\gamma$ " is a deeply bound molecular channel in all cases. The nonadiabatic couplings are sketched in Fig. 1 and indicate the regions where inelastic transitions are most likely. From our numerical calculations and on physical grounds, we believe that Fig. 11 represent all three-body systems near threshold.

Knowing the dependence of the potentials and couplings on $R$ and $a$ enables us to derive the scaling laws from the definitions of the rates,

$$
V_{\text {rel }} \propto\left|T_{f i}\right|^{2} / k \text { and } K_{3} \propto\left|T_{f i}\right|^{2} / k^{4},
$$

in terms of the transition probabilities $\left|T_{f i}\right|^{2}$. Only the wave vector important for the threshold law has been included $-k^{2}=2 \mu\left(E-E_{v l^{\prime}}\right)$ for $V_{\text {rel }}$ and $k^{2}=2 \mu E$ for $K_{3}$. The transitions proceed via tunneling in the initial potential to the $R$ where the coupling peaks. The transition 

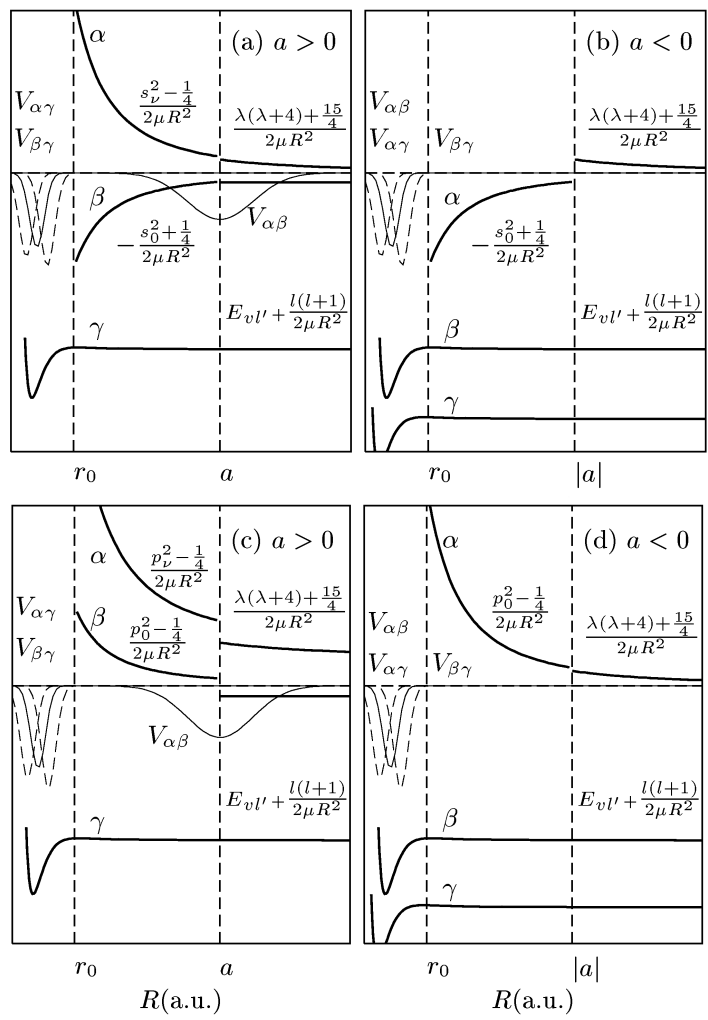

FIG. 1: Schematic plot of the three-body effective potentials for $0^{+}$bosonic systems for (a) $a>0$ and (b) $a<0$, and for the others systems, for (c) $a>0$ and (d) $a<0$.

probability can thus be approximated by the WKB tunneling probability (including the Langer correction [1]]),

$$
P_{x \rightarrow y}^{(\nu)} \approx \exp \left[-2 \int_{y}^{x} \sqrt{2 \mu\left(W_{\nu}(R)+\frac{1 / 4}{2 \mu R^{2}}-E\right)} d R\right] .
$$

The Langer correction is crucial for obtaining the correct scaling with $E$ and $a$, and introduces "tunneling" even when there is no barrier in $W_{\nu}$. At ultracold temperatures, the classical turning point is much larger than $|a|$.

In all cases, the couplings peak at $R \approx|a|, R \approx r_{0}$, or both. Relaxation for $a>0$, for instance, only occurs at small distances, $R \approx r_{0}$, where the coupling peaks $\left[V_{\beta \gamma}\right.$ in Figs. 1(a) and 1(c)]. It follows that the tunneling probability in the initial channel $\beta$ must be evaluated by integrating Eq. (7) from the classical turning point $r_{c}$ to $r_{0}$. This range, however, spans both kinds of potentials so that the tunneling in the two regions must be included,

$$
\left|T_{\gamma \beta}\right|^{2} \simeq P_{r_{c} \rightarrow a}^{(\beta)} P_{a \rightarrow r_{0}}^{(\beta)} .
$$

Similarly, the transition probability for recombination must also include tunneling in two different regions between $r_{c}$ and $r_{0}$. Recombination for $a>0$ can occur at two distances, though $-R \approx a$ and $R \approx r_{0}$ - since the coupling peaks both places, leading to

$$
\left|T_{\beta \alpha}\right|^{2} \simeq P_{r_{c} \rightarrow a}^{(\alpha)}+P_{r_{c} \rightarrow a}^{(\alpha)} P_{a \rightarrow r_{0}}^{(\beta)}+P_{r_{c} \rightarrow a}^{(\alpha)} P_{a \rightarrow r_{0}}^{(\alpha)}
$$

where each term corresponds to a different reaction pathway. Although not indicated here, these paths can interfere and will be discussed below. For $a<0$, all couplings peak at small distances $R \approx r_{0}$. Applying the arguments above, relaxation and recombination are, respectively,

$$
\left|T_{\gamma \beta}\right|^{2} \simeq P_{r_{c} \rightarrow r_{0}}^{(\beta)} \quad \text { and } \quad\left|T_{\beta \alpha}\right|^{2} \simeq P_{r_{c} \rightarrow|a|}^{(\alpha)} P_{|a| \rightarrow r_{0}}^{(\alpha)} .
$$

We can now determine both the threshold laws and the scattering length scaling. Since $W_{\nu}$ has the same form in the region $R>|a|$ in all cases [Eq. (3)], the tunneling probabilities in this region are also the same,

$$
P_{r_{c} \rightarrow|a|}^{(\alpha)} \propto(k a)^{2 \lambda+4} \text { and } P_{r_{c} \rightarrow|a|}^{(\beta)} \propto(k a)^{2 l+1} .
$$

Equation (11) completely determines the threshold laws for each process [2]. The scaling with $a$, however, will be strongly modified by the tunneling probability in the region $r_{0}<R<|a|$ due to Efimov physics.

For $0^{+}$bosons with $a>0$, the $\beta$-channel potential is attractive in the region $r_{0}<R<a$. Consequently, the probability $P_{a \rightarrow r_{0}}^{(\beta)}$ [Eqs. (8) and (9)] is not a tunneling probability, but rather a transmission probability that can be determined from general arguments based on the known solutions for Eq. (4) [6, 9]. This analysis gives

$$
V_{\text {rel }} \propto\left[\frac{\sinh (2 \eta)}{\sin ^{2}\left[s_{0} \ln \left(a / r_{0}\right)+\Phi\right]+\sinh ^{2}(\eta)}\right] a,
$$

since $l=0$ gives the leading contribution in Eq. (11). The constant $\eta$ is related to the probability for transitions at small distances [6] and $\Phi$ is an unknown small- $R$ phase. Equation (12) was deduced in Ref. 6], generalizing the result presented in Ref. [9] by letting the transition probability at small distances be less than unity. In both cases, however, the linear dependence on $a$ was obtained by dimensional and physical arguments, while it follows directly from Eq. (11) in the present analysis.

For $0^{+}$bosons with $a<0$, the initial state for relaxation is a deeply bound vibrational state independent of $a$. The transmission probability - and thus the relaxation rate — does not depend on $a$ either, leading to

$$
V_{\mathrm{rel}} \propto A_{\eta} k^{2 l} r_{0}^{2 l+1} .
$$

The constant $A_{\eta}$ will generically represent small- $R$ physics that can give resonances due to a three-body Feshbach resonance, but is otherwise independent of $a$.

For all cases other than $0^{+}$bosons, the initial channel for relaxation is never attractive in the region $r_{0}<R<|a|$ [Figs. 1(c) and 1(d)] so that relaxation proceeds by tunneling only. For $a>0$, the relaxation rate is

$$
V_{\text {rel }} \propto A_{\eta} k^{2 l}\left(\frac{r_{0}}{a}\right)^{2 p_{0}} a^{2 l+1} .
$$

The rate thus decreases with $a$ whenever $2 l+1<2 p_{0}$, yielding longer molecular lifetimes for larger $a$. For the specific case of a weakly bound $\mathrm{FF}^{\prime} s$-wave molecule, 


\begin{tabular}{c|c|ccc|ccc}
\hline \hline & & \multicolumn{4}{|c}{$V_{\text {rel }}$} & \multicolumn{4}{c}{$K_{3}\left(D_{3}\right)$} \\
& $J^{\pi}$ & $E$ & $a>0$ & $a<0$ & $E$ & $a>0$ & $a<0$ \\
\hline $\mathrm{BBB}$ & $0^{+}$ & const & $\boldsymbol{a}$ & const & const $\left(\boldsymbol{k}^{\mathbf{4}}\right)$ & $\boldsymbol{a}^{\mathbf{4}}$ & $|\boldsymbol{a}|^{4}$ \\
& $1^{-}$ & $k^{2}$ & $a^{-2.728}$ & const & $k^{6}\left(k^{10}\right)$ & $a^{10}$ & $|a|^{4.272}$ \\
& $2^{+}$ & $k^{4}$ & $a^{-0.647}$ & const & $k^{4}\left(k^{8}\right)$ & $a^{8}$ & $|a|^{2.353}$ \\
\hline $\mathrm{BBB}^{\prime}$ & $0^{+}$ & const & $\boldsymbol{a}$ & const & const $\left(\boldsymbol{k}^{\mathbf{4}}\right)$ & $\boldsymbol{a}^{4}$ & $|\boldsymbol{a}|^{4}$ \\
& $1^{-}$ & $k^{2}$ & $a^{-1.558}$ & const & $k^{2}\left(k^{6}\right)$ & $a^{6}$ & $|a|^{1.443}$ \\
\hline $\mathrm{FFF}^{\prime}$ & $0^{+}$ & const & $\boldsymbol{a}^{-3.332}$ & const & $k^{4}\left(k^{8}\right)$ & $a^{8}$ & $|a|^{3.668}$ \\
& $1^{-}$ & $k^{2}$ & $a^{-0.546}$ & const & $\boldsymbol{k}^{\mathbf{2}}\left(\boldsymbol{k}^{\mathbf{6}}\right)$ & $\boldsymbol{a}^{\mathbf{6}}$ & $|\boldsymbol{a}|^{2.455}$ \\
& $2^{+}$ & $k^{4}$ & $a^{-1.210}$ & const & $k^{4}\left(k^{8}\right)$ & $a^{8}$ & $|a|^{1.790}$ \\
\hline \hline
\end{tabular}

TABLE II: Threshold and scattering length scaling laws for three-body rates. Boldface indicates dominant contributions.

$p_{0}=2.1662220$ so that $V_{\text {rel }} \propto a^{-3.332444}$. This result agrees with the recent prediction of Petrov et al. 7] and is consistent with experiments [1]. Like the $0^{+}$boson systems, relaxation does not depend on $a$ for $a<0$,

$$
V_{\text {rel }} \propto A_{\eta} k^{2 l} r_{0}^{2 l+1} .
$$

The present analysis applies equally well to recombination. For instance, recombination of $0^{+}$bosons with $a>0$ is determined from Eq. (9), which includes three different recombination paths. Only the interference between the first two terms in Eq. (9) will be included here, since we expect the third term will be suppressed. In fact, this interference is well known [3, 4], and the present analysis reproduces the known expression plus a modification:

$$
K_{3} \propto\left[\sin ^{2}\left[s_{0} \ln \left(a / r_{0}\right)+\Phi\right]+A_{\eta}\left(\frac{r_{0}}{a}\right)^{2 s_{\nu}}\right] a^{4} .
$$

The first term in Eq. (16) is the usual result, while the second is due to recombination at small $R$. Because the small- $R$ coupling lies so far into the classically forbidden region, we expect $A_{\eta}$ to be small. For $a<0$, the present analysis yields the same expression found in Ref. [4],

$$
K_{3} \propto\left[\frac{\sinh (2 \eta)}{\sin ^{2}\left[s_{0} \ln \left(|a| / r_{0}\right)+\Phi\right]+\sinh ^{2}(\eta)}\right] a^{4} .
$$

The recombination rate for all other cases can be determined, giving for $a>0$ and $a<0$, respectively,

$$
\begin{gathered}
K_{3} \propto k^{2 \lambda}\left[1+A_{\eta}\left(\frac{r_{0}}{a}\right)^{2 p_{0}}+B_{\eta}\left(\frac{r_{0}}{a}\right)^{2 p_{\nu}}\right] a^{2 \lambda+4}, \\
K_{3} \propto k^{2 \lambda}\left(\frac{r_{0}}{|a|}\right)^{2 p_{0}}|a|^{2 \lambda+4},
\end{gathered}
$$

predicting an asymmetry in $K_{3}$ for $a<0$ and $a>0$. The constants $A_{\eta}$ and $B_{\eta}$ are expected to be small.

Our numerical results for $\mathrm{B}+\mathrm{BB}$ and $\mathrm{F}+\mathrm{FF}^{\prime}$ collisions confirm Eqs. (12) - (15). In fact, in our model for $\mathrm{F}+\mathrm{FF}^{\prime}$ the contributions for both $s$ - and $p$-wave final states scale as $a^{-3.33}$, emphasizing that the scaling only depends on the initial state. Equations (16)-(18) have been verified numerically and their limitations studied [5].
Table main power-law behavior of each rate. The two dominant partial waves, determined by their energy dependence, are shown for each process. For completeness, we include the scaling laws for the dissociation rate $D_{3}$ (for which $k^{2}=2 \mu E$ ). It is interesting to note that for $\mathrm{F}+\mathrm{FF}^{\prime}$ collisions where the relaxation rate decreases with $a$, the dissociation rate grows (see Table III) and will eventually become the dominant mechanism of molecular loss. The table also indicates the competition from the next leading term for finite temperatures. For fermion relaxation, this term is comparatively more important than for boson relaxation.

In this Letter, we have deduced the scaling laws for ultracold three-body collision rates for all symmetries. We have used a simple and intuitive approach that describes all three-body collision processes in the same framework. For relaxation and recombination, the scaling laws depend only on the initial state. Any dependence on the final state is expected to enter via the coupling terms, and should at most be weakly dependent on energy and scattering length. The present results apply only in the threshold regime, i.e. when the collision energy is the smallest energy in the system [5]. We have also shown the remarkable influence of Efimov physics - even for systems without bosons.

This work was supported by the National Science Foundation and by the Research Corporation.

[1] M. Greiner, C. A. Regal, and D. Jin, Nature (London) 426, 537 (2003); S. Jochim et al., Science 302 2101 (2003); M. W. Zwierlein et al., Phys. Rev. Lett. 92, 120403 (2004); J. Cubizolles et al., ibid. 91, 240401 (2003); K. E. Strecker, G. B. Partridge, and R. G. Hulet, ibid., 91080406 (2003).

[2] B.D. Esry, C.H. Greene, and H. Suno, Phys. Rev. A 65, R010705 (2002).

[3] B. D. Esry, C. H. Greene, and J. P. Burke, Phys. Rev. Lett. 83, 1751 (1999); E. Nielsen and J. H. Macek, ibid. 83, 1566 (1999).

[4] P. F. Bedaque, E. Braaten, and H. -W. Hammer, Phys. Rev. Lett. 85, 908 (2000); E. Braaten and H. -W. Hammer, ibid. 87, 160407 (2001).

[5] J. P. D'Incao, H. Suno, and B. D. Esry, Phys. Rev. Lett 93, 123201 (2004).

[6] E. Braaten and H.-W. Hammer, Phys. Rev. A 70, 042706 (2004).

[7] D. S. Petrov, C. Salomon, and G. V. Shlyapnikov, Phys. Rev. Lett. 93, 090404 (2004).

[8] H. Suno, B. D. Esry, C. H. Greene and J. P. Burke, Jr., Phys. Rev. A 65, 042725 (2002).

[9] V. Efimov, Sov. J. Nucl. Phys. 12, 589 (1971); 29, 546 (1979); Nucl. Phys. A210, 157 (1973).

[10] E. Nielsen, D. V. Fedorov, A. S. Jensen, and E. Garrido, Phys. Rep. 347, 373 (2001).

[11] R. E. Langer, Phys. Rev. 51, 669 (1937). 